\title{
Emetine regulates the alternative splicing of caspase 9 in tumor cells
}

\author{
DANMIN PAN ${ }^{1}$, KRITSANAPOL BOON-UNGE $^{2,3}$, PIYARAT GOVITRAPONG ${ }^{2}$ and JIANHUAZHOU ${ }^{1,3}$ \\ ${ }^{1}$ JiangSu Key Laboratory of Neuroregeneration, Nantong University, Nantong, Jiangsu 226001, P.R. China; \\ ${ }^{2}$ Neuro-Behavioural Biology Center, Mahidol University, Salaya, Nakornpathom 73170, Thailand; \\ ${ }^{3}$ Department of Medicine, Umass Medical School, Worcester, MA 01605, USA
}

Received April 11, 2011; Accepted August 17, 2011

DOI: $10.3892 / \mathrm{ol} .2011 .395$

\begin{abstract}
Exons 3 to 6 in the caspase 9 gene undergo alternative splicing in which the larger caspase 9 splice variant promotes apoptosis, in contrast to the dominant negative anti-apoptotic splice variant, the smaller caspase $9 \mathrm{~b}$. In this study, the regulation of the alternative splicing of caspase 9 pre-mRNA was examined in response to Emetine. Treatment of C33A cells, breast cancer MCF-7 cells and MCF-7/Adr cells with Emetine dihydrochloride upregulated the level of smaller caspase $9 \mathrm{~b}$ mRNA and concomitantly decreased the mRNA level of larger caspase 9 in a dose- and time-dependent manner, indicating that Emetine desensitizes C33A, MCF-7 and MCF-7/Adr to cell death. In contrast, treatment of PC3 cells, a prostate cancer cell line, manifested an opposite effect: a greater production of the larger caspase 9 mRNA with a concomitant decrease of caspase $9 \mathrm{~b}$ mRNA. Pretreatment with calyculin A, an inhibitor of protein phosphatase 1 (PP1) and protein phosphatase 2A (PP2A) blocked Emetine-induced alternative splicing in cells, in contrast to okadaic acid, a specific inhibitor of PP2A, demonstrating a PP1-mediated mechanism. These results suggest that the various splicing patterns of the caspase 9 gene that are regulated by chemotherapy reagents may contribute to the resistance or sensitization of the tumors to other cell death inducers.
\end{abstract}

\section{Introduction}

Apoptosis is a common and well-understood mechanism of cell death resulting from the activation of caspases through two primary pathways: i) via caspase 9 activation and a mitochondrial route to release cytochrome $\mathrm{c}$, or ii) directly via caspase 8 to caspase 3 as a primary effector caspase. At least 14 caspases have been identified and characterized in mammalian cells. Caspases fall into two classes: initiator caspases (caspases 8, 9,2 and 10) that exclusively cleave and activate

Correspondence to: Professor Jianhua Zhou, Jiangsu Key Laboratory of Neuroregeneration, Nantong University, 19 Qixiu Road, Nantong, Jiangsu 226001, P.R. China

E-mail: jianhua55@msn.com

Key words: alternative splicing, Emetine, caspase 9 other caspases, and effector caspases (caspases 3,6 and 7) that cleave other proteins. Procaspases are activated by cleavage at the same consensus site, either by themselves or by other caspases. Other proteins targeted by caspases include inhibitory apoptotic proteins (IAPs), inhibitors of caspase-activated DNAase (ICADs), Bcl-2, signal pathway proteins, cytoskeletal proteins and DNA repair enzymes, including DNA-dependent protein kinases. These different target proteins of caspase result in apoptotic cell death. It has been shown more recently that one of the mechanisms of regulating the balance between proliferation and cell death is through alternative splicing of apoptotic genes. Deregulation of this balance represents a pro-tumorigenic principle or neuronal death in human carcinogenesis or neurodegenerative diseases (1).

Splicing is a cellular process that removes introns from the pre-mRNA in eukaryotic genomes so that exons join together to form a mature mRNA. However, when the introns of a certain pre-mRNA are excised in more than one way, alternative splicing occurs, resulting in several possible mature mRNAs from one gene. Alternative pre-mRNA splicing is an essential mechanism for generating protein diversity (2-5). It is estimated that more than $60 \%$ of human genes undergo alternative splicing, leading to the production of diversified functional isoforms (6). Alternative splicing is precisely regulated. Aberrant splicing may lead to human disorders, including growth hormone deficiency, spinal muscular atrophy $(7,8)$ and possibly tumors and neurodegenerations.

A number of pre-mRNAs for cell death signals, including caspase 9, one of the most significant factors in the apoptotic pathways, are alternatively spliced, yielding isoforms with opposing functions during programmed cell death (8-10). Findings of various reports demonstrated that the alternative splicing of caspase 9 can be regulated by small molecules such as ceramide in cancer cells (11), as well as by other biological molecules SRp30a (ASF/SF2), E2F and SC35 $(12,13)$. To identify additional small molecules that are potentially used in cancer treatment to regulate caspase 9 splicing, we performed reverse transcriptase-polymerase chain reaction (RT-PCR) experiments in cells treated with 1,040 FDA-approved drugs and compounds. Emetine, a potent protein synthesis inhibitor in eukaryotes (14), was found to downregulate pro-apoptotic caspase 9 and upregulate anti-apoptotic caspase $9 \mathrm{~b}$ in $\mathrm{C} 33 \mathrm{~A}$ and MCF-7 cells, while in PC3 cells, an opposite effect was 
observed: caspase 9b was downregulated and caspase 9 was upregulated. We further demonstrated that this Emetine function was mediated by phosphorylation (15). However, the opposite effect of Emetine on caspase 9 splicing in C33A and PC 3 cells suggests that targeting alternative splicing for cancer treatment should be cautiously assessed.

\section{Materials and methods}

Compounds. All chemicals including Emetine, calyculin A and okadaic acid were purchased from Sigma (St. Louis, MO, USA).

Cell culture. The human cervical carcinoma C33A cells were maintained in Dulbecco's modified Eagle's medium (DMEM), supplemented with $10 \%$ (vol/vol) fetal bovine serum (FBS), L-Glutamine and 1X penicillin-streptomycin. PC3 prostate cancer cells were cultured in RPMI supplemented with $10 \%$ (v/v) FBS, L-Glutamine and $1 \mathrm{X}$ penicillin-streptomycin. Human breast cancer cells MCF-7 and MCF-7/Adr were cultured in RPMI supplemented with 10\% (v/v) FBS, L-Glutamine and 1X penicillin-streptomycin. All cells were maintained at less than $80 \%$ confluence under standard incubator conditions.

Emetine treatment. Emetine dihydrochloride hydrate (Sigma) with a stock solution concentration of $100 \mu \mathrm{M}$ was used. Twenty-four hours prior to Emetine treatment, the cells were plated in $2 \mathrm{ml}$ medium in 6-well plates at a density of 200,000 cells/well. The cells were treated with various concentrations of Emetine for $24 \mathrm{~h}$ for a dose-dependent study. For the time course experiment, cells were treated with $1.0 \mu \mathrm{M}$ Emetine for various durations.

Protein phosphatase inhibitor treatment. Cells were pretreated with calyculin A or okadaic acid for $1 \mathrm{~h}$. The media were removed. Fresh regular media with Emetine were added to treat cells for the duration of $24 \mathrm{~h}$. RT-PCR was then carried out to examine caspase 9 splicing.

Reverse transcriptase-polymerase chain reaction. Total RNA was extracted from cultured cells using TRIzol reagent (Invitrogen Corp., Carlsbad, CA, USA) according to the manufacturer's instructions. Reverse transcription was carried out with $1 \mu \mathrm{g}$ total RNA using Improm II reverse transcriptase (Promega, Madison, WI, USA) and oligo (dT) as the priming agent. Following incubation for $1 \mathrm{~h}$ at $42^{\circ} \mathrm{C}$, the reactions were terminated by heating at $70^{\circ} \mathrm{C}$ for $15 \mathrm{~min}$. To analyze alternative splicing of the caspase 9 gene, an upstream $5^{\prime}$ primer (5'-GCTCTTCCTTTGTTCATCTCC-3') and a downstream 3' primer (5'-CATCTGGCTCGGGGTTACTGC-3') were used for PCR amplification $\left(35\right.$ cycles at $94^{\circ} \mathrm{C}, 30 \mathrm{sec} ; 55^{\circ} \mathrm{C}, 30$ sec; $72^{\circ} \mathrm{C}, 1 \mathrm{~min}$ ) with Choice Taq Blue Mastermix (Denville Scientific Inc., South Plainfield, NJ, USA). PCR products were separated and analyzed on agarose gels.

This study was approved by the ethics committee at Nantong University, Nantong, Jiangsu, China.

\section{Results}

Emetine regulates the alternative splicing of caspase 9 pre-mRNA. To identify small molecules that regulate alterna-
$\mathbf{A}$

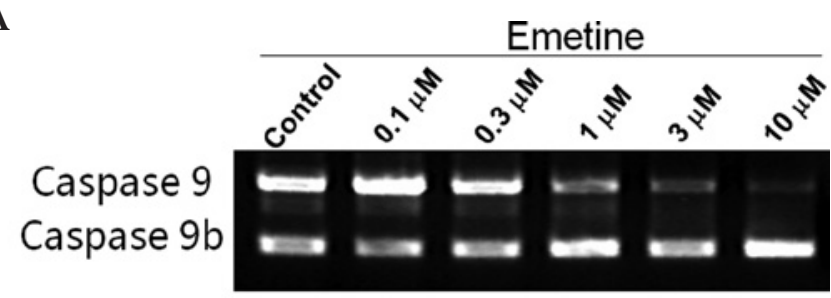

B

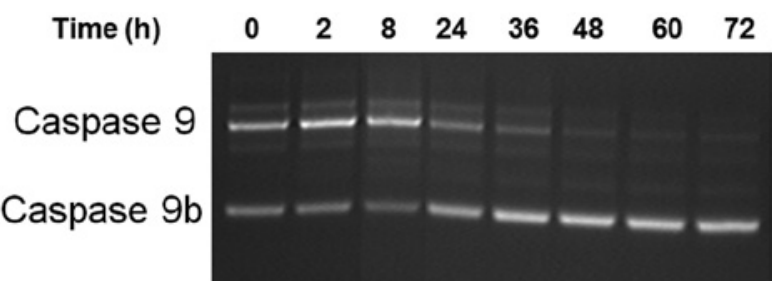

C

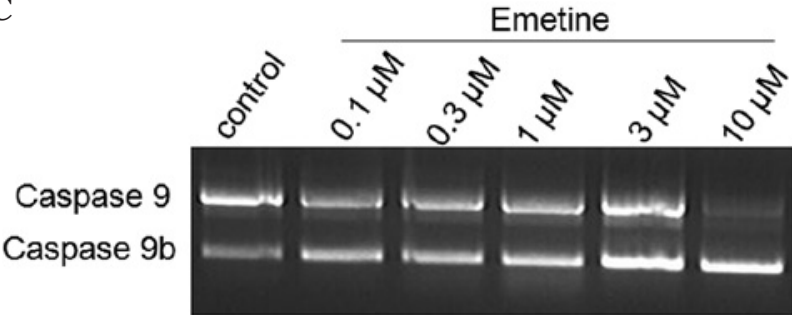

D

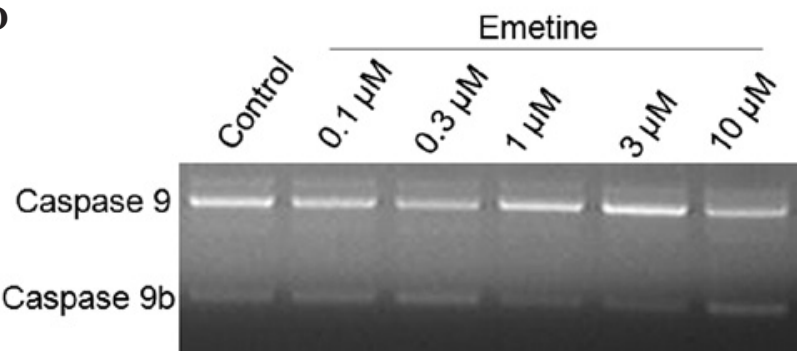

Figure 1.Emetine regulates caspase 9 splicing in C33A cells. C33A cells were treated with Emetine. Total RNA was extracted and analyzed by RT-PCR for the alternative splicing of caspase 9. Alternative splicing of the caspase 9 gene produced larger caspase 9 and smaller caspase $9 \mathrm{~b}$. The decrease of caspase 9 and increase of caspase $9 b$ was correlated with the (A) Emetine concentration and the (B) time duration. (C) MCF-7 and (D) MCF-7/Adr breast cancer cells were also treated with various concentrations of Emetine. Emetine regulated splicing of caspase 9 in these cells in a similar pattern as in $\mathrm{C} 33 \mathrm{~A}$ cells, although less pronounced.

tive splicing, 1,040 FDA-approved drugs and compounds were screened using RT-PCR in C33A cells, a cervical cancer line. As described in our previous study, Emetine regulated alternative splicing of the $B c l-x$ gene (15). Notably, Emetine was also found to increase the smaller caspase $9 \mathrm{~b}$ mRNA with a concomitant increase of the larger caspase 9 mRNA (Fig. 1). To further validate our findings, C33A cells were treated with various concentrations of Emetine or for different time durations. A decrease in the ratio of caspase 9/ caspase $9 \mathrm{~b}$ was observed (Fig. 1A). In our previous studies, Emetine was also found to alter splicing of the $B c l-x$ gene (15) but not the $t a u, S M N$ or BACEl genes, indicating its relative specificity on caspase 9 and Bcl-x splicing. To further validate whether Emetine regulates splicing in the caspase 9 gene and to evaluate whether regulation of caspase 9 splicing has potential relevance to cancer therapy, we examined the 
A

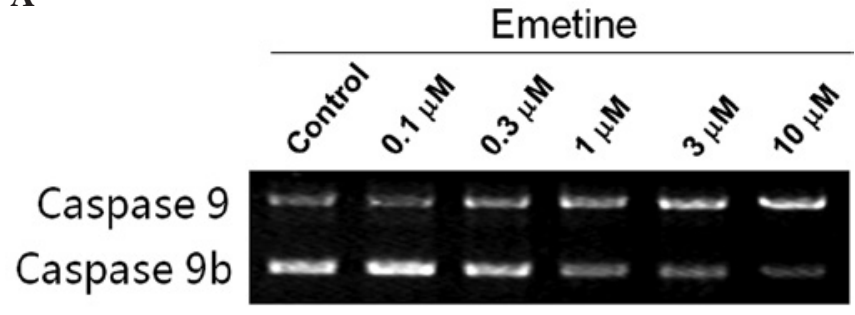

B

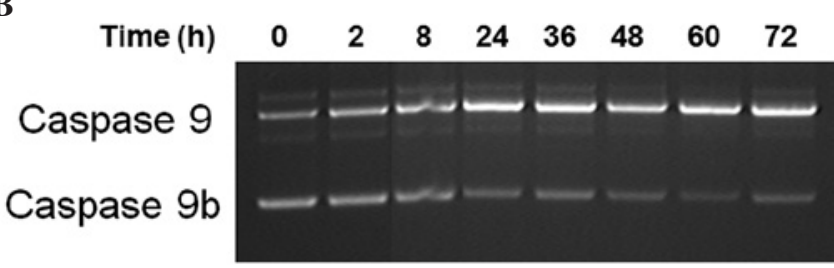

Figure 2. Emetine regulates caspase 9 splicing in PC3 cells. PC3 cells were treated with Emetine. Total RNA was extracted and analyzed by RT-PCR for the alternative splicing of caspase 9. Alternative splicing of the caspase 9 gene in PC 3 cells produced the larger caspase 9 and the smaller caspase $9 \mathrm{~b}$ However, in contrast to the results in C33A, Emetine was found to decrease caspase $9 \mathrm{~b}$ and increase caspase 9 . The results were correlated with the (A) Emetine concentration and the (B) time duration.

effects of Emetine on the pre-mRNA processing of caspase 9 in several tumor cell lines. Cells were treated over various time durations and/or with various concentrations of Emetine. Semi-quantitative RT-PCR was used to determine the effects of Emetine. In MCF-7 and MCF-7/Adr cells (breast cancer cell lines) (Fig. 1C and D), regulation of caspase 9 splicing was found to be slightly less pronounced than in C33A cells. However, the pattern of the regulation was similar to that observed in C33A cells; i.e., an increase of caspase $9 \mathrm{~b}$ and a decrease of caspase 9. Conversely, in PC3 (a prostate cancer cell line) cells (Fig. 2), an opposite effect of Emetine on caspase 9 splicing was noted: an increase of caspase 9 and a decrease of caspase $9 \mathrm{~b}$. The effects of Emetine on the pre-mRNA processing of caspase 9 in PC 3 cells were time course- and dose-dependent (Fig. 2A and B).

Emetine exerts its effect on caspase 9 splicing, via protein phosphatase 1 (PPI). Previous studies showed that ceramide and Emetine affect the splicing of $B c l-x$ in a phosphorylationdependent pathway. To examine whether Emetine exerts the effects on caspase 9 splicing in a similar manner, C33A and PC3 cells were treated with phosphatase inhibitors calyculin $\mathrm{A}$ and okadaic acid. We found that $5 \mu \mathrm{M}$ calyculin $\mathrm{A}$, an inhibitor of both protein phosphatase 1 (PP1) and protein phosphatase 2A (PP2A), blocked the Emetine effects on caspase 9 alternative splicing in $\mathrm{C} 33 \mathrm{~A}$ and $\mathrm{PC} 3$ cells (Fig. 3, comparing + Emetine with +Emetine/calyculin A). To establish whether PP1 or PP2A was the Emetine-responsive protein phosphatase that regulates caspase 9 alternative splicing, C33A and PC3 cells were pretreated for $1 \mathrm{~h}$ with $5 \mu \mathrm{M}$ okadaic acid, a selective PP2A inhibitor. Pretreatment with okadaic acid had no effect on caspase 9 alternative splicing (Fig. 3, comparing + Emetine with +Emetine/okadaic acid). Taken together, these results suggest that PP1 mediates the effects of Emetine on the alternative splicing of caspase 9 .
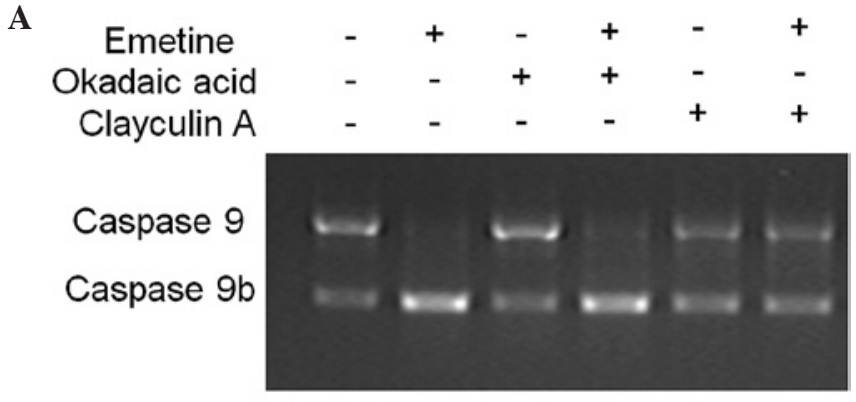

$\mathbf{B}$

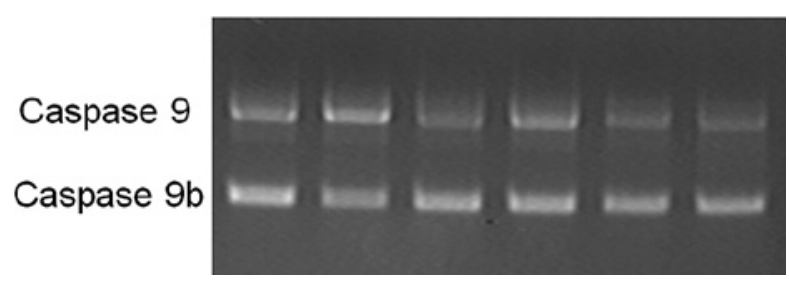

Figure 3. Calyculin A, but not okadaic acid, blocks the effect of Emetine on caspase 9 splicing. Cells were pretreated with either $5 \mu \mathrm{M}$ calyculin A, an inhibitor of both protein phosphatase 1 (PP1) and protein phosphatase 2A (PP2A), or with $5 \mu \mathrm{M}$ okadaic acid, a selective PP2A inhibitor, and then exposed to $1.0 \mu \mathrm{M}$ Emetine for $24 \mathrm{~h}$. RT-PCR was carried out. The results suggest that PP1, but not PP2A, mediates the effects of Emetine on the alternative splicing of caspase 9. (A) Effects of calyculin A in C33A cells (B) Effects of calyculin A in PC3 cells. All experiments were repeated at least three times. +, with Emetine, okadaic acid or calyculin A; -, without Emetine, okadaic acid or calyculin A.

\section{Discussion}

We previously described that Emetine regulated alternative splicing of Bcl-x, increasing the smaller pro-apoptotic Bcl-xS isoform and decreasing the larger anti-apoptotic Bcl-xL isoform, resulting in the possible sensitization of tumor cells to chemotherapy (15). We further demonstrated that Emetine modulated Bcl-x splicing by affecting PP1 phosphatase. These results were consistent with an earlier study (11) that a second chemotherapy agent, ceramide, also affected Bcl-x splicing through PP1. Notably, the authors of that study also demonstrated that in addition to Bcl-x, ceramide regulated alternative splicing of the caspase 9 gene through a similar mechanism, leading to an increase of the larger pro-apoptotic caspase 9 and a decrease of the smaller anti-apoptotic caspase 9b. Based on their studies, it appears that there is a synergetic effect on splicing of Bcl-x and caspase 9 in A549 cells, leading to an increase of pro-apoptotic isoforms for the two genes and to sensitization of A549 cells to death inducers.

However, in contrast to the effects of ceramide on the splicing of Bcl-x and caspase 9 in A549 cells, i.e., an increase of the pro-apoptotic forms of $B c l-x(\mathrm{Bcl}-\mathrm{xS})$ and caspase 9 (caspase 9) genes, this study revealed that Emetine has an opposite effect on the alternative splicing of caspase 9 in different tumor cell lines. In PC3 cells, Emetine increased pro-apoptotic caspase 9 (full-length) with a concomitant decrease of anti-apoptotic smaller caspase 9b, while in C33A and MCF-7 cells Emetine increased anti-apoptotic caspase $9 \mathrm{~b}$ with a decrease of the full-length pro-apoptotic caspase 9 (Figs. 1-3). The results in C33A and MCF-7 cells were unexpected, as Emetine had been considered to be one of the potential chemotherapeutic agents assumed to induce cell 
death. As discussed in our earlier study (15), Emetine acts as an effective chemotherapeutic agent by increasing the lifespan of tumor-bearing mice $(16,17)$ and thus has the possibility for clinical advantage $(18,19)$. However, although no previous study explains the mechanism responsible for the anti-tumor effect of Emetine, our results regarding the effects of Emetine on Bcl-x splicing (15) suggested that Emetine promoted the expression of pro-apoptotic proteins by regulating alternative splicing. However, based on the results in this study, we deduce that Emetine sensitizes cells to chemotherapy in certain types of tumors but does not sensitize them in others by regulating alternative splicing of caspase 9 in various ways. It is possible that a balance exists among apoptotic genes including $\mathrm{Bcl}-\mathrm{x}$ and caspase 9 . In addition, it appears that chemotherapy that is effective for one type of cancer may not be effective for others, although the target is the same (such as caspase 9). Our studies provide insights into the future treatment of cancers by targeting alternative splicing.

\section{Acknowledgements}

Dr Kritsanapol Boon-Unge was partly funded by the Royal Thai Government Staff Development Scholarship. JZ was funded by a Nantong University start-up fund and by the Priority Academic Program Development of Jiangsu Higher Education Institution (PAPD).

\section{References}

1. Mercatante D and Kole R: Modification of alternative splicing pathways as a potential approach to chemotherapy. Pharmacol Ther 85: 237-243, 2000.

2. Black DL: Protein diversity from alternative splicing: a challenge for bioinformatics and post-genome biology. Cell 103: 367-370, 2000.

3. Graveley BR: Alternative splicing: increasing diversity in the proteomic world. Trends Genet 17: 100-107, 2001.

4. Goldstrohm AC, Greenleaf AL and Garcia-Blanco MA: Co-transcriptional splicing of pre-messenger RNAs: considerations for the mechanism of alternative splicing. Gene 277: 31-47, 2001.
5. Caceres JF and Kornblihtt AR: Alternative splicing: multiple control mechanisms and involvement in human disease. Trends Genet 18: 186-193, 2002.

6. Modrek B and Lee C: A genomic view of alternative splicing. Nat Genet 30: 13-19, 2002.

7. Krawczak M, Reiss J and Cooper DN: The mutational spectrum of single base-pair substitutions in mRNA splice junctions of human genes: causes and consequences. Hum Genet 90: 41-54, 1992.

8. Schwerk C and Schulze-Osthoff K: Regulation of apoptosis by alternative pre-mRNA splicing. Mol Cell 19: 1-13, 2005.

9. Johnson CR and Jarvis WD: Caspase-9 regulation: an update. Apoptosis 9: 423-427, 2004.

10. Moore MJ, Wang Q, Kennedy CJ and Silver PA: An alternative splicing network links cell-cycle control to apoptosis. Cell 142: 625-636,

11. Chalfant CE, Rathman K, Pinkerman RL, et al: De novo ceramide regulates the alternative splicing of caspase 9 and $\mathrm{Bcl}-\mathrm{x}$ in A549 lung adenocarcinoma cells. Dependence on protein phosphatase-1. J Biol Chem 277: 12587-12595, 2002.

12. Massiello A and Chalfant CE: SRp30a (ASF/SF2) regulates the alternative splicing of caspase-9 pre-mRNA and is required for ceramide-responsiveness. J Lipid Res 47: 892-897, 2006.

13. Merdzhanova G, Edmond V, De Seranno S, et al: E2F1 controls alternative splicing pattern of genes involved in apoptosis through upregulation of the splicing factor SC35. Cell Death Differ 15: 1815-1823, 2008.

14. Grollman AP: Inhibitors of protein biosynthesis. V. Effects of emetine on protein and nucleic acid biosynthesis in HeLa cells. J Biol Chem 243: 4089-4094, 1968.

15. Boon-Unge K, Yu Q, Zou T, Zhou A, Govitrapong P and Zhou J: Emetine regulates the alternative splicing of Bcl-x through a protein phosphatase 1-dependent mechanism. Chem Biol 14: 1386-1392, 2007.

16. Jondorf WR, Abbott BJ, Greenberg NH and Mead JA: Increased lifespan of leukemic mice treated with drugs related to (-)-emetine. Chemotherapy 16: 109-129, 1971.

17. Johnson RK and Jondorf WR: Some inhibitory effects of (--)emetine on growth of Ehrlich ascites carcinoma. Biochem J 140: 87-94, 1974 .

18. Panettiere F and Coltman CA Jr: Experience with emetine hydrochloride (NSC 33669) as an antitumor agent. Cancer 27: 835-841, 1971.

19. Siddiqui S, Firat D and Olshin S: Phase II study of emetine (NSC-33669) in the treatment of solid tumors. Cancer Chemother Rep 57: 423-428, 1973. 